\title{
Virtual games and quality of life in Parkinson's disease: A randomised controlled trial
}

\author{
Glicia Pedreira, Antonio Prazeres, Danilo Cruz, Irênio Gomes, Larissa Monteiro*, Ailton Melo
}

Department of Neuroscience and Mental Health, Faculty of Medicine, Federal University of Bahia, Salvador, Brazil;

*Corresponding Author: menezes.lari@gmail.com

Received 27 March 2013; revised 25 May 2013; accepted 5 June 2013

Copyright (C) 2013 Glicia Pedreira et al. This is an open access article distributed under the Creative Commons Attribution License, which permits unrestricted use, distribution, and reproduction in any medium, provided the original work is properly cited.

\begin{abstract}
Objective: To evaluate the efficacy of Nintendo Wii training in quality of life in Parkinson's disease (PD) patients when compared to traditional physical therapy (PT). Methods: A randomized, single-blinded trial with 2 parallel arms was performed in a referral center for movement disorders in North-eastern, Brazil. Forty-four PD outpatients that fulfilled the eligibility criteria with mild to moderate motor impairment were randomized. Both groups executed a warm up session for $\mathbf{1 0}$ minutes that consisted of trunk flexion, extension and rotation, associated with upper and lower limbs stretching. The PT group followed a program that consisted of trunk and limb mobilisation, balance, muscle strengthening, rhythmic movement, postural alignment, double-task execution, bimanual tasks, and gait training. The Nintendo Wii group executed a sequence of tasks according to a previously established protocol, with similar training exercises. Duration of exercises was $\mathbf{4 0}$ minutes per session, 3 days per week for 4 weeks. The primary endpoint was the total score obtained in the Parkinson's disease quality of life questionnaire (PDQ-39) translated from English to Brazilian Portuguese by Oxford outcomes. Secondary endpoints were the scores achieved by each group in the following domains of PDQ-39 scale: mobility, activities of daily living (ADL), emotional well-being, stigma, social support, cognition, communication and bodily discomfort. Assessments were performed before and after intervention in both groups with subjects in the "on" period. Results: Subjects in the Nintendo Wii group showed greater improvement in the PDQ-39 total score when compared to PT
\end{abstract}

group $(p=0.01)$. Also, significant differences were observed in ADL, stigma, social support and communication when comparing subjects before and after intervention in the Nintendo Wii group $(p<0.05)$. Conclusions: The results achieved in this trial suggest that rehabilitation using Nintendo Wii may have beneficial effects in quality of life of PD subjects, when compared to traditional PT. Further larger randomised controlled-trials are necessary to reassure these results.

Keywords: Parkinson's Disease; Rehabilitation; Nintendo Wii; Quality of Life; Physical Therapy

\section{INTRODUCTION}

Parkinson's disease (PD) is classically characterised by rigidity, tremor, bradykinesia, and postural instability [1]. However, non-motor symptoms such as fatigue, autonomic dysfunctions, impaired mood and cognition increase the risk of frequent fallings, social isolation and decreased quality of life [2]. Although the relevance of traditional physical therapy (PT) has been recognized to alleviate PD symptoms and improve quality of life, there are no clear guidelines or practical uniform recommendations that support this treatment. Furthermore, some patients have limited access to physical therapy facilities, lowering the motivation to maintain prescribed exercise programs. Virtual games (VG) such as Nintendo Wii have some advantages over PT. The games are less expensive and portable. In addition, they reproduce wellknown sports and may help to develop motor and cognitive skills.

The efficacy of virtual rehabilitation in functional performance of patients with poststroke paresis, spinal cord injury, and cerebral palsy has been widely demonstrated $[3,4]$. The use of VG in poststroke patients has 
demonstrated improvements in active and passive movements, as indicated by the Wolf Motor Function Test and Fugl-Meyer Assessment scores [5]. However, despite several case reports showing motor improvements in PD patients after exercising with VG, no clear evidence has been presented so far to show whether these exercises are beneficial to improve quality of life in PD patients.

The objective of this trial was to evaluate the effects of Nintendo Wii training in quality of life of PD patients, when compared to traditional PT.

\section{METHODS}

\subsection{Study Design}

This study was a randomized, single-blinded trial with parallel groups and pretest/posttest assessments.

\subsection{Ethical Approval, Consent, and Registration}

Informed consent forms, patients information, study protocol, questionnaires and exercises procedures were approved by the Ethics Committee at Federal University of Bahia. The Helsinki declaration was followed in every procedure, and this study was registered at clinicaltrials.gov (NCT01120392).

\subsection{Study Participants and Recruitment}

Subjects with diagnosis of PD by a certified neurologist according to the United Kingdom Brain Bank Criteria with mild to moderate motor impairment (stages 1 - 3 on Hoehn and Yahr scale), aged between 45 and 80 years, were invited to participate in the study. Patients who fulfilled the inclusion criteria were enrolled from July to October 2011 in an referral center of Movement Disorders at Federal University of Bahia, North-eastern, Brazil. Exclusion criteria were dementia, uncontrolled hypertension, heart disease, psychiatric disorders, and illnesses that prevented exercise understanding and performance.

\subsection{Study Procedures}

Before performing trial procedures, subjects were instructed to: 1) mobilise their trunks and execute flexion, extension and rotation movements; 2) stretch their upper and lower limbs and maintain each sequence for $60 \mathrm{~s}$ over a 10-minute period. This warm up was followed by a 40-minute physical therapy program that consisted of PT or Nintendo Wii ${ }^{\circledR}$ VG. The traditional exercises addressed trunk and limb mobilisation, balance, muscle strengthening, rhythmic movement, postural alignment, double-task execution, bimanual tasks, and cardiorespiratory and gait training. The Nintendo $\mathrm{Wii}^{\circledR} \mathrm{VG}$ and exercises are listed in Figure $\mathbf{1}$ according to their objectives. During the Nintendo $\mathrm{Wii}^{{ }^{\circledR}}$ game sessions, the pa-

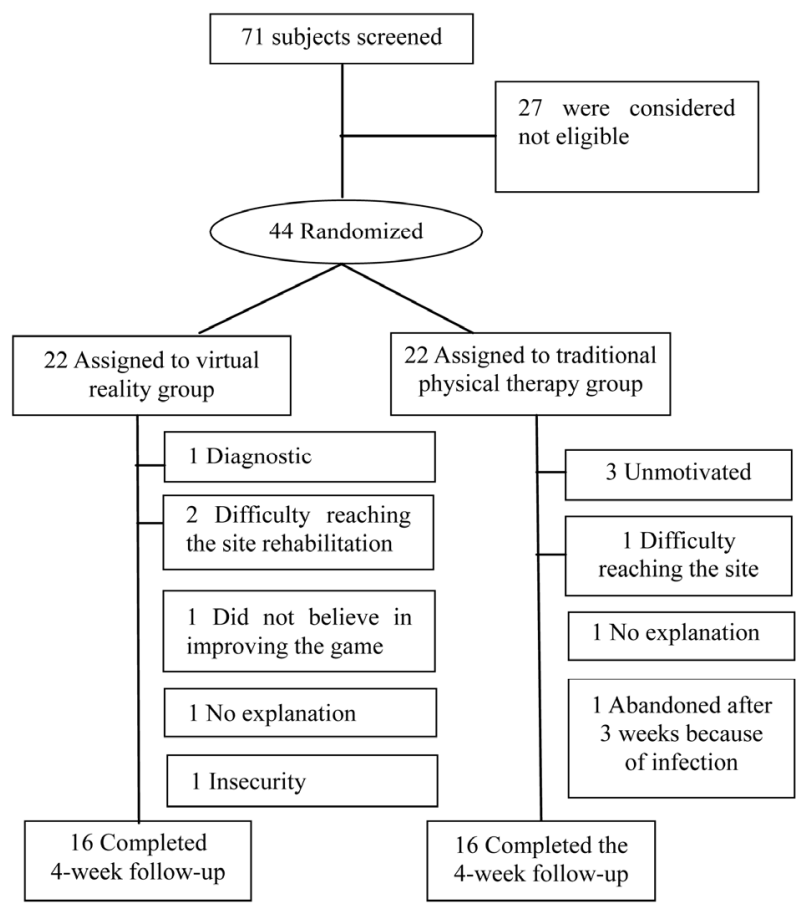

Figure 1. Flow diagram with study procedures according to CONSORT statement.

tients paused for 1 minute before changing games. The first week was used to familiarise the patients with the exercises and games. The exercises in both groups were performed 3 times weekly for 1 month (12 sessions).

The virtual rehabilitation exercises were conducted in a $20 \mathrm{~m}^{2}$ room that was equipped with a Nintendo $\mathrm{Wii}^{\circledR}$, and a projector hanging from the ceiling. The image was projected onto the wall at a height of $160 \mathrm{~cm}$ to stimulate an erect posture during the exercises. Nintendo Wii is a home videogame with several exercises that allow training on strength, balance and aerobics. Also, players are stimulated to defeat their adversaries in specific trainings like boxing, and can track their progress over time, enhancing adhesion and concentration in their physical activity.

An occupational therapist who had specific training for this purpose and who was blinded to the other parts of the research applied the Unified Parkinson's Disease Rating Scale (UPDRS) and the 39-item PD quality of life scale (PDQ-39) validated to Brazilian Portuguese [6]. Assessments were performed either at baseline and 4 weeks after the intervention. The evaluations and interventions were performed 1 hour after administering levodopa, during the "on" period.

\subsection{Randomisation}

Patients were assigned to either the Nintendo $\mathrm{Wii}^{\circledR}$ group or the PT group based on computerised randomisation. 


\subsection{Statistical Analysis}

An independent and blinded statistician performed the statistical analysis with SPSS version 17.0. To compare groups, the Pearson $\chi^{2}$ test or Fisher test of frequencies and the Mann-Whitney $U$ test for quantitative data were used. The Wilcoxon signed rank test was used for withingroup before and after treatment comparisons. Results were considered positive if an improvement of $10 \%$ or more on the scale used was achieved. Data were considered significant at $P<0.05$.

\section{RESULTS}

A total of 71 subjects were recruited and 44 that fulfilled the eligibility criteria were randomized to traditional PT or Nintendo Wii, with the same number of patients in each arm (Figure 1). Baseline characteristics of the population studied showed no significant differences in age, gender, disease duration, and disease stage between groups (Table 1). The UPDRS scores ranged from 14 to 71, and the Hoehn and Yahr varied from 1 to 2.5.

At baseline, the PDQ-39 scores showed impaired quality of life for all PD patients. After the intervention, all patients submitted to Nintendo Wii training showed an improvement of more than $10 \%$ in their total quality of life scores $(P<0.05)$ (data not shown). When analyzing specific domains of PDQ-39, subjects in Nintendo Wii group showed significant improvement in social support, communication, ability to deal with stigma and perform daily living activities (Table 2). No differences in the outcomes analyzed were observed in the group submitted to traditional PT.

\section{DISCUSSION}

The results of this trial suggest that rehabilitation of

Table 1. Baseline characteristics of studied subjects.

\begin{tabular}{cccc}
\hline & Nintendo Wii & PT & P \\
\hline Gender (\%) & $11(68.8)$ & $11(73.3)$ & $1.000^{*}$ \\
Male & $5(31.3)$ & $4(26.7)$ & \\
Female & & & \\
Clinical predominance (\%) & $10(66.7)$ & $9(60.0)$ & 0.705 \\
Tremor & $5(33.3)$ & $6(40.0)$ & \\
Rigidity & & & \\
Screening age (y) & $61.1 \pm 8.2$ & $66.2 \pm 8.5$ & 0.105 \\
& & & \\
Disease duration (y) & & & \\
& $8.6 \pm 4.6$ & $7.3 \pm 6.6$ & 0.541 \\
Hoehn and Yahr score at baseline & & & \\
& $2.5 \pm 0.6$ & $2.4 \pm 0.7$ & 0.572 \\
\hline
\end{tabular}

PT = Physical Therapy.
Table 2. Comparison of PDQ-39 scores within and between groups after intervention (traditional physical therapy or Nintendo Wii).

\begin{tabular}{|c|c|c|}
\hline & Wii & PT \\
\hline \multicolumn{3}{|l|}{ Mobility } \\
\hline before & $36.69 \pm 25.28$ & $20.5 \pm 23.23$ \\
\hline after & $27.94 \pm 23.75$ & $21.17 \pm 21.4$ \\
\hline$P$ & 0.064 & 0.812 \\
\hline difference & $-8.75 \pm 17.94$ & $0.67 \pm 9.7$ \\
\hline \multicolumn{3}{|l|}{ ADL } \\
\hline before & $35.19 \pm 24.42$ & $21.94 \pm 18.6$ \\
\hline after & $26.38 \pm 22.88$ & $22.5 \pm 27.49$ \\
\hline$P$ & 0.009 & 0.789 \\
\hline difference & $-8.81 \pm 9.89$ & $0.56 \pm 19.53$ \\
\hline \multicolumn{3}{|c|}{ Emotional well-being } \\
\hline before & $39.88 \pm 35.25$ & $21.95 \pm 17.85$ \\
\hline after & $30.25 \pm 28.12$ & $29.45 \pm 20.14$ \\
\hline$P$ & 0.056 & 0.068 \\
\hline difference & $-9.63 \pm 19.08$ & $7.5 \pm 14.87$ \\
\hline \multicolumn{3}{|l|}{ Stigma } \\
\hline before & $29.44 \pm 28.46$ & $15 \pm 19.16$ \\
\hline after & $15.31 \pm 19.22$ & $15 \pm 18.72$ \\
\hline$P$ & 0.021 & 0.931 \\
\hline difference & $-14.13 \pm 20.87$ & $0 \pm 21.78$ \\
\hline \multicolumn{3}{|c|}{ Social Support } \\
\hline before & $18.19 \pm 22.9$ & $9.44 \pm 19.12$ \\
\hline after & $8.31 \pm 16.31$ & $3.33 \pm 7.59$ \\
\hline$P$ & 0.018 & 0.144 \\
\hline difference & $-9.88 \pm 17.59$ & $-6.11 \pm 17.95$ \\
\hline \multicolumn{3}{|l|}{ Cognition } \\
\hline before & $36.81 \pm 25.42$ & $25.42 \pm 24.6$ \\
\hline after & $26.63 \pm 18.36$ & $25 \pm 21.91$ \\
\hline$P$ & 0.053 & 0.857 \\
\hline difference & $-10.19 \pm 23.08$ & $-0.42 \pm 16.61$ \\
\hline \multicolumn{3}{|c|}{ Communication } \\
\hline before & $26 \pm 19.14$ & $17.22 \pm 15.58$ \\
\hline after & $17.25 \pm 14.88$ & $13.89 \pm 14.66$ \\
\hline$P$ & 0.009 & 0.476 \\
\hline difference & $-8.75 \pm 11.39$ & $-3.33 \pm 14.37$ \\
\hline \multicolumn{3}{|c|}{ Bodily discomfort } \\
\hline Before & $45.19 \pm 25.76$ & $23.89 \pm 23.75$ \\
\hline after & $38.44 \pm 23.86$ & $27.22 \pm 23.88$ \\
\hline$P$ & 0.396 & 0.623 \\
\hline difference & $-6.75 \pm 29.97$ & $3.33 \pm 23.74$ \\
\hline \multicolumn{3}{|l|}{ TOTAL } \\
\hline before & $34.25 \pm 18.61$ & $19.95 \pm 13.98$ \\
\hline after & $24.23 \pm 16$ & $21.83 \pm 15.05$ \\
\hline$P$ & 0.012 & 0.733 \\
\hline difference & $-9.14 \pm 12.43$ & $1.88 \pm 9.37$ \\
\hline
\end{tabular}

$P=p$ value. 
PD patients with Nintendo $\mathrm{Wii}^{\circledR}$ improves quality of life through several aspects such as communication abilities, social support, stigma and activities of daily living. There were no significant differences in the group submitted to traditional PT.

PD patients exhibit several factors that can negatively influence their quality of life, such as functional limitations, low self-esteem, social restriction, reduction of leisure time, and functional dependency [7]. In this trial, we observed that the utilisation of physical therapy strategies based on Nintendo Wii virtual games improved almost all of the test-related elements in most patients.

Virtual reality promoted by Nintendo Wii games simulates a real environment with visual, auditory, and perceptual feedback that positively influences motor learning and quality of movements, particularly in PD patients. Two recently published articles found that the use of virtual reality goggles resulted in greater task improvements, completion time and gait performance when compared to patients exercising without the goggles $[8,9]$.

Cognition is another important factor in motor learning, development, and quality of life. An improvement in cognitive functions, as demonstrated by the PDQ-39 specific domain, was observed in this study. Virtual games also contain cognitive tasks related to attention and executive functions, which are also affected in PD patients [10].

A high degree of participant interactivity and satisfaction was observed during Nintendo Wii games execution. As we observed, patient motivation for task execution is an important factor for motor, cognitive, and learning performance. This motivation may have led to a greater adherence to the treatment when compared with studies of physical therapy without virtual reality [3].

To our knowledge, this is the first study that demonstrates the effects Nintendo Wii virtual games on the quality of life in PD patients.

\section{LIMITATIONS}

This study had several limitations. The main limitation of this study was the impossibility to blind patients to the procedure performed. As a consequence, some patients randomly assigned to the traditional physical therapy group have demonstrated their frustration. To overcome this problem, before entering the study, all patients were informed that they could migrate to their exercise of choice after 1 month in any group. All patients (except for 4) in the traditional PT group have migrated to Nintendo Wii group, and the degree of adherence has been high thus far. In this way, the results point the possibility of new rehabilitation strategies that can highly improve quality of life in PD patients.

\section{CONCLUSION}

This trial suggests that rehabilitation using Nintendo $\mathrm{Wii}^{\circledR}$ is superior to traditional PT to improve quality of life in PD patients.

\section{AKNOWLEDGEMENTS}

This study was supported by the Brazilian National Institutes of Science and Technology (CITECS-MCT-CNPq)

\section{REFERENCES}

[1] Poewe, W. (2008) Non-motor symptoms in Parkinson's disease. European Journal of Neurology, 15, 14-20. http://dx.doi.org/10.1111/j.1468-1331.2008.02056.x

[2] Keus, S.H., Bloem, B.R., Hendriks, E.J., Bredero-Cohen, A.B., Munneke, M. and Practice Recommendations Development Group (2007) Evidence-based analysis of physical therapy in Parkinson's disease with recommendations for practice and research. Movement Disorders, 22, 451-460. http://dx.doi.org/10.1002/mds.21244

[3] Albani, G., Pignatti, R., Bertella, L., Priano, L., Semenza, C. and Molinari, E. (2002) Common daily activities in the virtual environment: A preliminary study in Parkinsonian patients. Neurological Sciences, 23, S49-S50. http://dx.doi.org/10.1007/s100720200064

[4] Saposnik, G., Teasell, R., Mamdani, M., Hall, J., McIlroy, W., Cheung, D., et al. (2010) Effectiveness of virtual reality using Wii gaming technology in stroke rehabilitation: A pilot randomized clinical trial and proof of principle. Stroke, 41, 1477-1484.

http://dx.doi.org/10.1161/STROKEAHA.110.584979

[5] Mouawad, M.R., Doust, C.G., Max, M.D. and McNulty, P.A. (2011) Wii-based movement therapy to promote improved upperextremity function post-stroke: A pilot study. Journal of Rehabilitation Medicine, 43, 527-533. http://dx.doi.org/10.2340/16501977-0816

[6] Jenkinson, C., Fitzpatrick, R., Peto, V., Greenhall, R. and Hyman, N. (1997) The Parkinson's Disease Questionnaire (PDQ-39): Development and validation of a Parkinson's disease Summary Index Score. Ageing, 26, 353-357.

[7] Koepp, M.J., Gunn, R.N., Lawrence, A.D., Cunningham, V.J., Dagher, A., Jones, T., et al. (1998) Evidence for striatal dopamine release during a video game. Nature, 393, 266-268.

[8] Griffin, H.J., Greenlaw, R., Limousin, P., Bhatia, K., Quinn, N.P. and Jahanshahi, M. (2011) The effect of real and virtual visual cues on walking in Parkinson's disease. Journal of Neurology, 258, 991-1000.

[9] Mirelman, A., Maidan, I., Herman, T., Deutsch, J.E., Giladi, N. and Hausdorff, J.M. (2011) Virtual reality for gait training: Can it induce motor learning to enhance complex walking and reduce fall risk in patients with Parkinson's disease? Journals of Gerontology Series A: Biological Sciences and Medical Sciences, 66, 234-240. http://dx.doi.org/10.1093/gerona/glq201

[10] Cherniack, E.P. (2011) Not just fun and games: Applica- 
tions of virtual reality in the identification and rehabilitation of cognitive disorders of the elderly. Disability and
Rehabilitation: Assistive Technology, 6, 283-289. http://dx.doi.org/10.3109/17483107.2010.542570 